\title{
Climate-smart agriculture : pour une agriculture climato-compatible
}

\author{
Emmanuel Torquebiau ${ }^{1,2, *}$ \\ ${ }^{1}$ CIRAD, UPR AIDA, 34398 Montpellier, France \\ 2 AIDA, Univ Montpellier, CIRAD, Montpellier, France
}

\begin{abstract}
Résumé - L'agriculture intelligente face au climat (climate-smart agriculture-CSA) a comme objectifs d'être adaptée au changement climatique et de l'atténuer, tout en contribuant de manière durable à la sécurité alimentaire. Né en 2010 à l'initiative de la FAO, le concept a fait école et se décline désormais en diverses pratiques qui prennent en compte ces objectifs de manière différente. Les pratiques agroécologiques de couverture permanente du sol, par des arbres ou des cultures, sont parmi les plus courantes. Mais la CSA prétend aussi être une approche plus large permettant de répondre aux enjeux du changement climatique par des politiques publiques et des financements innovants. Défini par des objectifs et non pas par les moyens d'atteindre ces objectifs, le concept de CSA pose aussi question, notamment sur la possibilité d'assurer simultanément ses trois objectifs et sur le risque d'encourager un productivisme déguisé qui ne respecterait ni l'environnement ni les agriculteurs. L'initiative « $4 \%$, les sols pour la sécurité alimentaire et le climat » reprend les objectifs de la CSA en insistant sur l'atténuation du changement climatique par l'augmentation de la teneur en carbone du sol. Tout en étant un concept qui demande encore à faire ses preuves, la CSA est une approche de l'agriculture originale et compatible avec les enjeux du changement climatique.
\end{abstract}

Mots clés : adaptation / atténuation / changement climatique / sécurité alimentaire / sol

Abstract - Climate-smart agriculture: making agriculture climate-friendly. The objectives of climatesmart agriculture (CSA) are to be adapted to climate change and mitigate it, while sustainably contributing to food security. The concept emerged in 2010 at FAO and has now become popular. Different CSA practices can be found today and integrate its objectives differently. Agroecological practices aiming for a permanent soil cover, either with trees or crops, are among the most common CSA practices. CSA is also a wider approach allowing taking into account the challenge of climate change with innovative public policies and financing. Because it is defined by its objectives and not by the means to reach those objectives, the concept of CSA has also been questioned, especially regarding the possibility to reach its three objectives simultaneously and because of the risks to promote disguised productivism that would not respect the environment or the farmers. The 4\% initiative «Soils for Food Security and Climate » takes up the objectives of CSA and emphasizes the mitigation of climate change by increasing the carbon content of the soil. While the CSA concept has yet to prove itself, it is an innovative, climate-friendly approach to agriculture that is compatible with the challenges of climate change.

Keywords: adaptation / mitigation / climate change / food security / soil

\section{Introduction}

La première définition précise du terme « climate-smart agriculture $»-\mathrm{CSA}$, Agriculture intelligente face au climat ou Agriculture climato-intelligente - apparaît en 2010 dans un

\footnotetext{
*Auteur de correspondance : emmanuel.torquebiau@cirad.fr
}

document de la FAO intitulé «Climate-Smart Agriculture » (FAO, 2010), avec le sous-titre suivant : «Policies, Practices and Financing for Food Security, Adaptation and Mitigation »-Politiques, pratiques et financements pour la sécurité alimentaire, l'adaptation et l'atténuation. On y trouve la définition suivante : "Agriculture that sustainably increases productivity, resilience (adaptation), reduces/removes greenhouse gases (mitigation), and enhances achievement of 
national food security and development goals $»-$ Agriculture qui augmente la productivité et la résilience (adaptation) des cultures de manière durable, favorise la réduction/élimination des gaz à effet de serre (atténuation), améliore la sécurité alimentaire nationale et contribue à la réalisation des objectifs de développement du pays (traduction en français dans FAO, 2011). L'expression « climate-smart » apparaît ici et là dans la littérature scientifique avant 2010, notamment dans le domaine forestier (ex : Nitschke et Innes, 2008), mais avec un sens plus général correspondant approximativement à : « prenant en compte les enjeux du changement climatique ». Les trois grands critères de CSA (adaptation, atténuation, sécurité alimentaire durable) sont connus comme les trois « piliers » de la CSA et leur satisfaction simultanée comme le « triplé gagnant » (triple win).

Le concept de CSA est né du constat que l'agriculture des pays en développement (cible géographique du document de la FAO) devait faire l'objet de transformations significatives pour répondre aux enjeux de la sécurité alimentaire et du changement climatique. Le sous-titre du document, en plaçant le mot «policies » en tête, signale clairement que c'est là que se situe l'enjeu principal. Cela est confirmé par le premier message-clé du document, qui indique que des pratiques climato-intelligentes sont déjà disponibles et peuvent donc être mises en œuvre sans attendre. Suivent 16 pages d'exemples (gestion $\mathrm{du}$ sol et des nutriments, systèmes semenciers, réduction des pertes post-récolte, agroforesterie, etc.) qui se concluent sur deux recommandations « transversales »:

- l'adoption d'une stratégie écosystémique, le travail à l'échelle du paysage et la coopération ou coordination intersectorielle sont fondamentaux pour réagir de façon efficace aux changements climatiques ;

- des investissements importants sont nécessaires pour compléter les données et connaissances manquantes, en matière de recherche et de développement de technologies.

Cette entrée en matière du concept de CSA met donc les choses au point et affirme que les «transformations significatives » qui sont nécessaires ne concernent pas que les pratiques agricoles, qui certes devront changer, mais aussi différents éléments de contexte de cette mise en œuvre. Le document de la FAO se poursuit sur la nécessité d'un appui au processus de CSA par des institutions renforcées, sur le besoin d'une meilleure cohérence entre les politiques agricoles, de sécurité alimentaire et de changement climatique, et enfin sur l'intérêt d'une synergie entre les financements privés et publics. On verra dans ce qui suit que cette mise au point initiale a son importance, dans la mesure où le concept de CSA a, par la suite, été interprété de différentes manières.

La FAO a présenté le concept de CSA à La Haye lors de la «Global Conference on Agriculture, Food Security and Climate Change » (31-10 au 5-11-2010) organisée par le gouvernement des Pays-Bas. C'est sans doute l'une des premières fois que les préoccupations concernant l'agriculture, la sécurité alimentaire et le changement climatique étaient réunies dans une même conférence, mais le projet FACCE-JPI (https://www.faccejpi.com/ Programmation conjointe de la Commission européenne ; site consulté le 28-07-2017), en date de décembre 2009, portait exactement le même nom (Agriculture, Food Security and Climate Change). La première de ces démarches était mondiale (Pays-Bas, Banque mondiale, FAO, CGIAR), plutôt orientée vers les pays du Sud, tandis que l'autre (FACCE-JPI) était centrée sur l'agriculture européenne. Toutes deux sont à rapprocher du processus qui a émergé à la même époque dans le cadre des négociations des Nations unies sur le changement climatique (UNFCCC). Si lors de la COP16 à Cancún en 2010, il était encore peu question d'agriculture, c'est à la COP17 à Durban, en 2011, que l'agriculture a été inscrite à l'ordre du jour des négociations sur le changement climatique (alors que les forêts y étaient depuis longtemps). Mais il faudra attendre l'accord de Paris en 2015 (COP21) pour que le mot " sécurité alimentaire " (mais toujours pas " agriculture »!) apparaisse dans un document officiel des négociations de l'UNFCCC. À la COP22 de Marrakech (novembre 2016), enfin, de nombreuses initiatives concernaient l'agriculture, par exemple, « 4 pour mille » (Les sols pour la sécurité alimentaire et le climat) ou "AAA » (Adaptation de l'agriculture africaine).

La conférence de La Haye s'est terminée sur une « feuille de route » recommandant de travailler simultanément sur :

- les politiques et les stratégies de la CSA ;

- les outils et techniques de la CSA ;

- les moyens financiers nécessaires au changement.

L'accent fut mis aussi sur la nécessité de faire passer le concept de CSA et ses déclinaisons dans les différentes instances internationales. À la suite de La Haye 2010, deux processus ont donc évolué en parallèle : un processus scientifique (conférences de Wageningen, Pays-Bas, en octobre 2011 et de Davis, Californie, en mars 2013, qui ont été suivies par la conférence de Montpellier en mars 2015) et un processus politique (conférence de Hanoï en septembre 2012 et conférence de Johannesburg en décembre 2013). Bien que ces deux processus soient séparés, la nécessité du lien entre science et politiques est demeurée une préoccupation permanente. Afin de maximiser l'impact de la démarche CSA, accélérer sa mise en œuvre et identifier des mécanismes de financement, les parties prenantes ont lancé, en septembre 2014 aux Nations unies à New York, la « Global Alliance on Climate-Smart Agriculture » (GACSA), une plate-forme ouverte dont l'objectif était de créer et catalyser des partenariats contribuant à une approche intégrée des trois piliers de la CSA (http://www.fao.org/gacsa/en/; site consulté le 28-07-2017). À l'heure où nous écrivons ces lignes, une quatrième conférence scientifique est prévue à Johannesburg en novembre 2017 (http://csa2017.nepad.org/en/ ; site consulté le 31-07-2017).

Face à une dynamique mondiale importante du concept de CSA, le présent article a pour objectif d'en préciser la définition et de présenter un point de vue sur les enjeux et les questions que pose ce concept dans le cadre des pays en développement. À la suite de quelques exemples concrets, nous montrons comment la CSA peut se décliner à différentes échelles spatiales allant de la parcelle au paysage. Nous insistons enfin sur la proximité conceptuelle entre la CSA et la récente initiative $4 \%$ sur le carbone du sol, qui ont toutes deux l'ambition de permettre à l'agriculture d'être " compatible » avec le changement climatique, en contribuant à son atténuation et en s'y adaptant pour contribuer à la sécurité alimentaire. 


\section{Qu'est-ce que la climate-smart agriculture?}

La CSA, depuis ses débuts, n'est pas définie comme une technique agronomique mais comme une approche permettant de répondre aux enjeux du changement climatique sur la base de solutions souples et adaptées au contexte, reposant sur des mécanismes politiques et financiers innovants (FAO, 2013 ; Lipper et al., 2014). Cette approche est censée permettre que les trois « piliers » (adaptation, atténuation, sécurité alimentaire durable) soient simultanément réunis. La logique réunissant ces trois paramètres dans une même démarche vient du fait que, face au changement climatique, l'agriculture est à la fois victime et responsable : victime, parce qu'elle subit les changements climatiques (hausse de la température, défaut ou excès d'eau, nouvelles maladies, etc.) ; responsable, parce qu'elle émet des gaz à effet de serre, dans des proportions non négligeables : $12 \%$ directement, et indirectement jusqu'à $24 \%$ (IPCC, 2014) si l'on inclut les changements d'utilisation des terres liés à l'exploitation forestière et aux fronts pionniers agricoles qui la suivent.

Mais l'agriculture fait aussi partie des solutions au problème du changement climatique puisqu'elle est capable, par le processus de la photosynthèse, d'augmenter le stock de carbone du sol aux dépens du gaz carbonique contenu dans l'atmosphère. À ce titre, l'agriculture apparaît comme une forme de géo-ingénierie « biologique » permettant de " décarboner » la planète, à côté de solutions beaucoup plus radicales comme le stockage de $\mathrm{CO}_{2}$ dans les couches géologiques. L'agriculture, en favorisant par des pratiques adaptées le stockage du carbone atmosphérique dans les sols, peut donc participer activement à l'atténuation du changement climatique et tendre vers la neutralité en gaz à effet de serre. De plus, ces pratiques vertueuses ont aussi des conséquences positives en termes de résilience et de propriétés physico-chimiques et biologiques du sol et conduisent à une meilleure adaptation de l'agriculture aux contraintes climatiques en augmentation, telles que l'irrégularité des saisons, les évènements extrêmes, l'augmentation de température, l'excès ou le défaut de pluie. La boucle est donc bouclée pour que l'agriculture devienne «compatible» avec les enjeux du changement climatique : si l'on associe atténuation des gaz à effet de serre et adaptation, on peut contribuer à de meilleurs rendements et à la sécurité alimentaire. Telle est la genèse du concept de CSA.

\section{Les questions que pose le concept de climate-smart agriculture}

L'atteinte simultanée des trois critères de la CSA est tout sauf simple. On peut imaginer une pratique consistant à produire beaucoup de biomasse, par exemple des plantes à croissance rapide destinées à produire de la bioénergie. C'est une solution d'atténuation du changement climatique (moindre utilisation d'énergie fossile, localement ou globalement). Elle peut contribuer à améliorer les revenus de ceux qui la pratiquent. Mais est-elle adaptée à tout environnement ? S'il est nécessaire, pour qu'elle fonctionne, d'amender le sol avec de grandes quantités d'engrais (émetteur de gaz à effet de serre), l'avantage d'atténuation sera perdu. Si cette solution est mise en œuvre aux dépens de terres agricoles consacrées à l'alimentation, l'avantage en termes de sécurité alimentaire sera remis en cause. Mais une telle stratégie peut aussi contribuer à l'adaptation si elle repose sur des ressources existantes, disponibles localement, ainsi qu'à la sécurité alimentaire en diminuant les dépenses liées à la consommation d'énergie (Tatsidjodoung et al., 2012). Dans ce cas, des mesures d'accompagnement semblent nécessaires en matière de technologies de production d'énergie adaptées à la dimension des exploitations et de gestion durable de la ressource (bois ou autre biomasse) et de l'impact potentiel de la production de bioénergie sur les surfaces consacrées à l'alimentation.

Les difficultés soulevées par cet exemple proviennent du fait que la définition de la CSA repose sur des objectifs à atteindre (les trois «piliers ») et non sur les moyens de les atteindre. Cette particularité a soulevé un certain nombre de critiques à l'endroit de la CSA, accusée parfois de ne pas être fondée sur une argumentation scientifique assez solide. La CSA a pu aussi être perçue comme une porte ouverte à toute forme d'agriculture ou de foresterie " productiviste », dont le seul objectif serait le rendement maximum, à n'importe quel coût économique ou environnemental, présentée fallacieusement comme le meilleur compromis atténuation-adaptation-sécurité alimentaire. Ce risque, et l'ouverture de la Global Alliance for Climate-Smart Agriculture (GACSA) au secteur agro-industriel privé, ont conduit un certain nombre d'organisations de la société civile à rédiger une lettre ouverte dénonçant le concept de CSA (http://www.climatesmartagconcerns.info/francais 1.html ; site consulté le 28-07-2017), qui s'opposerait aux principes de l'agroécologie.

Le concept de CSA a, en effet, été « récupéré » par un certain nombre de compagnies agroalimentaires qui s'en revendiquent sans respecter la définition d'origine. Mais il faut aussi rendre justice à cette définition centrée sur les objectifs qui permet - précisément parce que rien n'est dit sur la manière d'atteindre ces objectifs - que des moyens divers soient mis en œuvre, y compris ceux de l'agroécologie (Saj et al., 2017). Dit en d'autres termes, le concept de climatesmart agriculture est compatible aussi bien avec une vision de « modernisation écologique faible » (Weak ecological modernisation) que de « modernisation écologique forte » (Strong ecological modernisation) ; voir Horlings et Marsden, 2011. Les conditions sociales, économiques et environnementales de la mise en œuvre du concept de climate-smart agriculture doivent bien entendu être vérifiées (Caron et Treyer, 2015 ; Tissier et Grosclaude, 2015), mais le risque de dérive « productiviste » ne semble ni plus ni moins présent dans ce type d'agriculture que dans les autres formes de production agricole. Il est d'ailleurs exagéré d'écrire que rien n'est dit sur les moyens à mettre en œuvre pour atteindre les objectifs de la CSA. Dans le volumineux document publié par la FAO (2013), il est fait mention de nombreuses approches permettant d'atteindre les objectifs de la CSA, que ce soit dans le domaine des pratiques, des politiques publiques ou des institutions. La notion de résilience appliquée aux systèmes agricoles et l'utilisation durable des ressources (resource use efficiency) sont également citées fréquemment dans les documents fondateurs de la CSA (FAO, 2010 ; Lipper et al., 2014). L'agriculture intelligente face au climat ébranle, en effet, les frontières entre le monde de la recherche et celui des politiques publiques et le concept requiert encore d'être formalisé comme 
un cadre d'analyse et d'interprétation pour traiter des liens entre climat et agriculture (Caron, 2016).

Il est bien entendu également important que la CSA ne s'affranchisse pas d'une vision réfléchie de la sécurité alimentaire puisque celle-ci est bien partie intégrante de sa définition. La sécurité alimentaire existe lorsque tous les êtres humains ont, à tout moment, un accès physique et économique à une nourriture suffisante, saine et nutritive leur permettant de satisfaire leurs besoins énergétiques et leurs préférences alimentaires pour mener une vie saine et active (définition de la Conférence mondiale de l'alimentation, 1996). Premier point essentiel : ne pas interpréter la sécurité alimentaire comme une simple exigence quantitative de production, ce qui mènerait aux dérives productivistes dont on a parlé plus haut. La qualité nutritionnelle et l'accès régulier à une alimentation diversifiée sont des exigences tout autant essentielles, mais dans le contexte du changement climatique, d'autres exigences apparaissent, liées aux émissions de gaz à effet de serre ou à l'atténuation du changement climatique. La riziculture en submersion continue, la production industrielle de viande et de lait ou les échanges alimentaires internationaux (par exemple, le soja) sont à l'origine de fortes émissions de gaz à effet de serre (Garnett, 2011; Saunois et al., 2016) et ne peuvent prétendre à faire partie d'une approche CSA sans précautions particulières. Le drainage occasionnel des rizières (voir cidessous) contribue à diminuer les émissions de méthane. La gestion de l'élevage à l'échelle territoriale permet de réduire l'empreinte carbone de la viande (Vayssières et al., 2017). C'est avec des réserves de ce type que ces options peuvent être retenues dans le cadre de l'approche CSA. Ces exemples montrent aussi que des choix en termes de régime alimentaire sont susceptibles d'avoir un impact sur la mise en œuvre de la CSA, par exemple des régimes moins carnés ou qui ne dépendent pas de productions nécessitant de longs transports internationaux. De manière similaire, les options agroécologiques contribuant à augmenter la capture du carbone dans le sol ou la végétation seront naturellement susceptibles de mieux répondre à ces exigences climatiques (Saj et al., 2017).

On sait néanmoins que l'agroécologie est définie de différentes manières selon les auteurs et que le concept a évolué avec le temps, d'une vision environnementale de l'agriculture à l'écologie des systèmes alimentaires et à l'intégration de la souveraineté alimentaire (Wezel et al., 2009). L'agroécologie repose aussi sur la fourniture de services écosystémiques à partir de la biodiversité et restreint l'utilisation des intrants de synthèse exogènes à l'exploitation agricole. Ces critères peuvent être présents dans la CSA mais n'en sont pas une condition obligatoire. Cela a pu être à l'origine de certaines des critiques négatives sur la CSA.

\section{Exemples de climate-smart agriculture}

Prenons un autre exemple pour décrire l'atteinte simultanée des trois critères et tendre vers la compatibilité avec les enjeux du changement climatique. Pour augmenter le stock de matière organique du sol, on peut envisager qu'il soit couvert en permanence, y compris pendant la saison défavorable, sèche ou froide. Cet objectif peut être atteint par l'agroforesterie, en dispersant des arbres dans les parcelles de culture ou en cultivant sous un couvert arboré, ou bien par l'agriculture de conservation, en cessant le labour du sol et en associant à la culture principale une culture de couverture qui couvre le sol toute l'année. Ce sol protégé devient plus résilient face aux aléas climatiques (adaptation) et permet une augmentation du stock de carbone (atténuation). Si les cultures et les arbres sont bien choisis et les pratiques culturales envisagées compatibles avec les préférences des agriculteurs, on peut espérer que l'objectif de sécurité alimentaire soit atteint. De nombreux articles disponibles sur l'agriculture de conservation montrent néanmoins que ces principes sont souvent difficiles à atteindre, notamment dans les exploitations agricoles traditionnelles de la zone tropicale (Giller et al., 2015 ; Powlson et al., 2016).

D'autres exemples fournissent des éléments comparatifs. Des recherches en cours sur l'intensification de la riziculture irriguée en contexte d'agriculture familiale ont montré que l'on peut, avec des techniques d'irrigation intermittente, diminuer la consommation d'eau (adaptation), réduire les émissions de méthane dues à la décomposition anaérobique de la matière organique (atténuation) tout en améliorant les rendements ou la qualité (sécurité alimentaire) (Bouman et al., 2007 ; Joulian et al., 1997). Quelles sont les conditions supplémentaires pour que ces techniques passent dans l'usage ? Sans doute une sensibilisation à l'utilisation modérée des ressources en eau et au problème des émissions de gaz à effet de serre par les rizières, phénomène peu perçu par les agriculteurs dont les priorités quotidiennes sont ailleurs. À noter que l'absence d'immersion peut aussi poser des problèmes d'adventices, souvent mentionnés par les agriculteurs et pour l'instant mal résolus.

\section{La climate-smart agriculture à différentes échelles}

On trouve dans la CSA des options qui peuvent se décliner depuis l'échelle de la parcelle jusqu'à celle du paysage, comme, par exemple, la conservation et la gestion durable de l'eau et du sol : irrigation raisonnée, capture de l'eau, lutte contre l'érosion, enrichissement en matière organique, amélioration de la biodiversité du sol, cultures d'associations, plantes de couverture, composante arborée des champs et des paysages, etc. Une agriculture qui se préoccupe de son bilan carbone - notamment le stockage du carbone dans les parties aériennes et souterraines des plantes et dans le sol, ainsi que la réduction des émissions de gaz à effet de serre - imite les écosystèmes naturels de la région où elle se trouve plutôt que de tenter de modifier l'environnement à fortes doses d'engrais et d'irrigation. En zone de savane boisée, par exemple, une monoculture de céréales est un non-sens ; c'est une « agriculture pérenne » (Perfecto et al., 2009) qu'il faut encourager, fondée sur l'utilisation de ligneux, de plantes de couverture ou de graminées pérennes. En zone chaude et humide, les plantes à racines et tubercules, éventuellement cultivées sous ombrage, sont à privilégier. La CSA peut difficilement se résumer à une monoculture ou à une spécialisation et tend plutôt à l'intégration de différentes activités agricoles, par exemple, les exploitations associant agriculture et élevage, la pisciculture de rizière, l'agroforesterie. Si ces pratiques se rapprochent de certaines pratiques agroécologiques, elles s'en distinguent en ce sens que, pour 
pouvoir prétendre à « l'appellation » CSA, elles doivent nécessairement intégrer simultanément adaptation, atténuation et sécurité alimentaire. Bien que l'atténuation ne fasse pas partie des critères de l'agroécologie, il est évident que certaines démarches agroécologiques sont favorables à l'atténuation, par exemple, lorsque de grandes quantités de biomasse ou de matière organique sont produites.

La dimension paysage est particulièrement favorable à l'approche CSA car c'est l'échelle qui convient le mieux pour imaginer des mécanismes de synergie entre l'adaptation au changement climatique et son atténuation (Harvey et al., 2014). À cette échelle, la gestion des systèmes de production et des ressources naturelles couvre une superficie assez grande pour produire des services écosystémiques, mais assez petite pour être gérée par les personnes qui utilisent la terre qui produit ces services (FAO, 2013). Un des exemples récents les plus spectaculaires de CSA à cette échelle est celui dit du « reverdissement du Sahel », au Niger notamment (Reij et al., 2009). Des opérations de recherche-développement, la mise en place de la décentralisation et le transfert des droits de propriété des arbres de l'État aux paysans ont permis, dans ce pays, de relancer la pratique de la régénération naturelle assistée des arbres des champs. En quelques années, l'augmentation de la densité des arbres à l'hectare a été spectaculaire, contribuant à la modification du microclimat et de la fertilité du sol (adaptation), à l'augmentation de la biomasse sur pied (atténuation) et à l'amélioration des revenus et des conditions de subsistance des agriculteurs. Un des plus beaux exemples de compatibilité agriculture-changement climatique.

On peut donc parler de «climate-smart landscapes ». Ce sont des paysages « multifonctionnels » (Minang et al., 2015) caractérisés par une mosaïque ou une matrice dans laquelle apparaissent des unités reliées entre elles selon un réseau d'interactions biotiques et abiotiques, comme un mélange de champs, de pâturages, de zones naturelles, de parcelles boisées et de zones protégées. À l'intérieur de chacune de ces unités, on peut diversifier les cultures, les espèces et les itinéraires techniques, on peut recycler les composés organiques (fumiers, résidus d'émondage et ou de culture, utilisation des symbioses racinaires). Entre ces éléments de la mosaïque, on peut entretenir des haies et des ruisseaux de drainage, protéger des ripisylves (zones boisées le long des cours d'eau), installer des bandes herbeuses en périphérie des parcelles labourées, etc. Cette diversité spatiale peut se raisonner également au sein d'une exploitation agricole en décalant les dates de semis entre parcelles voisines, en mélangeant les parcelles ou en diversifiant les rotations, en installant des plantes mellifères, voire des ruches, ou encore en entretenant les haies comme dans un bocage. Ce partage d'objectifs de production et de protection de la nature dans le même espace est connu sous le nom de "land sharing" (partage de la terre) et s'oppose au « land sparing » (épargne de la terre) dans lequel on intensifie certaines zones et on poursuit des objectifs de protection de la nature dans d'autres espaces (Grau et al., 2013). Fausse bonne idée, le land sparing est à la base de la révolution verte et de l'agriculture industrielle des pays développés et n'a pas permis que s'accomplissent des objectifs de protection de la nature, tout en épuisant les sols agricoles.
Le territoire, paysage socialement construit, lieu privilégié de l'action collective, peut aussi être la cible d'actions répondant aux enjeux climatiques. On conçoit, en effet, qu'au niveau d'un village ou d'une région, la mise en œuvre d'une gestion de la terre répondant aux exigences du changement climatique nécessite que des propriétaires voisins, des collectivités territoriales, des agences de bassin ou de protection de la nature mènent des initiatives coordonnées. C'est ici que prend tout son sens l'effort «d'harmonie intersectorielle » recensé par la FAO comme faisant partie des conditions intégrantes de la CSA. Les secteurs en question sont l'agriculture, la forêt, la protection de la nature ou bien sûr la gestion de l'eau. Mais ce sont aussi les secteurs des finances publiques, des assurances, de l'aménagement du territoire, des travaux publics, de l'énergie, ou encore de la gestion des déchets. Enfin, le secteur privé est susceptible d'orienter les choses dans un sens ou dans l'autre par la loi de l'offre et de la demande et au moyen de partenariats avec le secteur public. Il est à noter que cette approche « territoire » est également promue par la convention désertification dans le cadre de la « dégradation neutre des terres » (http://www.csf-desertifica tion.org/combattre-la-desertification/item/fiche-la-neutraliteen-termes-de-degradation-des-terres ; site consulté le 28-072017).

\section{Le rôle du carbone du sol}

L'une des manières bien connues d'améliorer la performance environnementale de l'agriculture est de favoriser la matière organique $\mathrm{du}$ sol. Les chiffres montrent qu'une augmentation de $4 \%$ ( 4 pour mille ou $0,4 \%$ ) par an du stock de carbone organique dans les horizons de surface $(0-40 \mathrm{~cm})$ des sols de la planète permettrait de compenser l'ensemble des émissions nettes annuelles de gaz à effet de serre, à condition que l'on stoppe également la déforestation tropicale (Balesdent et Arrouays, 1999). En effet, les sols de la planète contiennent environ 1500 milliards de tonnes de carbone ( 2 à 3 fois plus que l'atmosphère), dont 860 milliards dans les premiers $40 \mathrm{~cm}$. Un apport de 3,4 milliards de tonnes de carbone par an aux 860 milliards de tonnes présents dans le sol correspond à environ $4 \%$. Ajoutés à une diminution des émissions de 0,9 milliard de tonnes en améliorant la gestion des zones forestières, cela permet d'équilibrer le bilan des émissions anthropiques annuelles de carbone, qui sont de 4,3 milliards de tonnes (Fig. 1).

C'est cette vision qui est à l'origine de l'initiative « $4 \%$ - Les sols pour la sécurité alimentaire et le climat » du Gouvernement français, dont le but est de promouvoir le stockage du carbone dans les sols agricoles et forestiers tout en assurant l'adaptation de l'agriculture au changement climatique et l'atteinte de la sécurité alimentaire. L'initiative comprend un plan d'action et un programme international de recherche dont l'ambition globale est de faire de l'agriculture l'une des solutions à la crise climatique (http://4p1000.org/ ; site consulté le 28-072017). Par ailleurs, de nombreux sols mal cultivés ou dégradés ont perdu leur matière organique et donc leur carbone et ne peuvent que bénéficier de cette initiative.

Une augmentation de $4 \%$ par an peut être obtenue par des pratiques telles que la couverture permanente du sol par des 

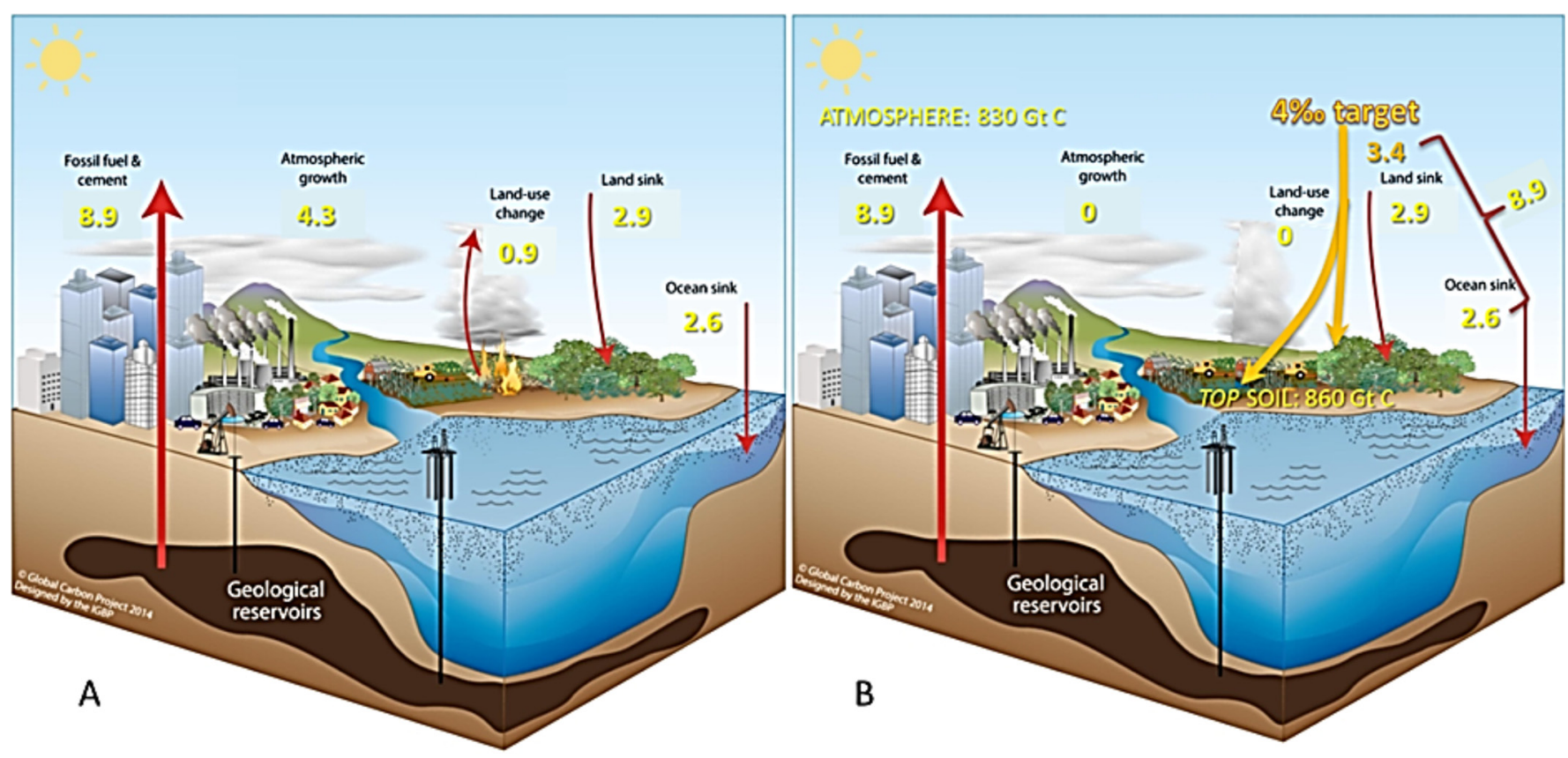

Fig. 1. Cycle du carbone avant (A) et après (B) mise en place d'une séquestration annuelle de carbone à hauteur de $4 \%$ du stock ( $0-40 \mathrm{~cm})$ des sols mondiaux.

(A) Perturbation du cycle global du carbone par les activités humaines en Gigatonnes (Gt) de carbone, en moyenne sur la décennie 2004-2013 (Gt C/an). Noter le rôle des 2 puits de carbone naturels que sont les continents et les océans (Le Quéré et al., 2015). (B) En stockant chaque année quatre millièmes du stock de carbone présent dans l'horizon de surface $(0-40 \mathrm{~cm})$ des sols mondiaux ( $860 \mathrm{Gt})$, le puits supplémentaire de carbone des continents augmenterait de 3,4 Gt de carbone par an. La somme des 3 puits de carbone (8,9 Gt de carbone par an) compenserait alors les émissions de $\mathrm{CO}_{2}$ issues de la combustion des énergies fossiles et de la production de ciment, à condition de stopper également les émissions nettes liées aux changements d'usage des sols (0,9 Gt de carbone par an).

Fig. 1. Carbon cycle before (A) and after (B) soil carbon sequestration at a rate of $4 \%$ of the carbon stock of world soils ( $0-40 \mathrm{~cm})$.

plantes annuelles ou des arbres, la restauration des sols dégradés, l'utilisation de légumineuses fixatrices de l'azote atmosphérique, l'emploi de fumier ou de compost ou encore les pratiques de collecte de l'eau de pluie ou de ruissellement (http://www.inra.fr/Grand-public/Rechauffement-climatique/ Tous-les-magazines/Quatre-pour-1000.-Stockage-du-car bone-dans-le-sol ; site consulté le 28-07-2017). L'hypothèse est ambitieuse mais semble réalisable et présente l'intérêt d'associer développement économique et réduction des gaz à effet de serre grâce aux « co-bénéfices » de l'atténuation en termes d'adaptation (Lal, 2016) ou vice-versa. On voit apparaître à nouveau la dimension de compatibilité entre une pratique et les contraintes du changement climatique. Il faut cependant remarquer que l'apport de carbone organique s'accompagne d'une perte par minéralisation et que l'augmentation du taux de carbone organique ne peut pas être indéfinie (Sommer et Bossio, 2014 ; Papy, 2016). D'autres questions de recherche se posent, comme, par exemple, la disponibilité en azote et en phosphore et la présence d'eau pour assurer un taux important de séquestration du carbone. La mise au point d'indicateurs fiables sur le taux de carbone du sol ou la promotion de politiques publiques susceptibles de convaincre les agriculteurs font également partie des thèmes importants à traiter. On notera bien sûr la proximité entre les concepts de CSA et de $4 \%$, le premier ciblant l'adaptation en priorité, le second l'atténuation. Mais les deux ciblant la sécurité alimentaire, la CSA de manière générale, le $4 \%$ par le biais du carbone du sol.

\section{Conclusion}

À condition d'en respecter la définition d'origine, la CSA peut représenter un authentique changement de paradigme contribuant à une approche intégrée de l'agriculture. Des politiques publiques fondées sur l'exigence de simultanéité entre les trois critères de la CSA auraient le mérite de conduire à un renouvellement de pratiques allant dans le sens d'une agriculture compatible avec les enjeux du changement climatique et capable de nourrir la planète. Mais il est sans doute utile d'insister sur le fait que l'approche CSA est complexe, liée au contexte, et ne peut être fondée sur des recettes toutes faites. Pour favoriser des synergies entre adaptation et atténuation, il faut diffuser de l'information à destination de tous les acteurs. Pour coordonner des secteurs qui s'ignorent, il faut une gouvernance innovante qui facilite la participation de tous. Pour s'accorder sur des objectifs de résilience, il faut analyser finement les conditions locales. Pour faciliter l'adoption d'innovations radicales telles que le $4 \%$, il faut que les politiques publiques, les financements et les institutions s'accordent. Pour qu'une approche climatiquement responsable fonctionne auprès des petits agriculteurs du Sud, il faut que l'adaptation au changement climatique devienne du « développement adaptatif », c'est-à-dire que l'amélioration de la sécurité alimentaire, du bien-être ou des revenus, fasse partie, par construction, de l'approche.

Mais les politiques publiques capables de répondre à cette complexité ne sont pas courantes et rien de ce qui précède ne se 
fera spontanément. Ni sous injonction, d'ailleurs. Il convient donc, pour promouvoir cette approche, de décrire à la fois les pratiques et les conditions supplémentaires permettant leur mise en œuvre afin qu'émerge un processus d'appropriation du changement. À défaut, les pratiques en question pourraient n'être que des options classiques d'agriculture durable déjà connues (cultures en courbe de niveau, gestion intégrée des bioagresseurs, rétention de l'eau, cultures d'associations, etc.) dont on sait le mal qu'elles ont parfois à être adoptées. Terminons en regrettant la terminologie retenue en français pour traduire climate-smart. "Climato-intelligent» ou « intelligent face au climat » pourraient laisser entendre que ce qui a précédé n'était pas intelligent... Le terme est, en effet, mal choisi et la notion de compatibilité avec le changement climatique évoquée dans le présent article pourrait être retenue pour renouveler la terminologie. Pourrait-on parler d'une agriculture « climato-compatible »?

Remerciements. L'auteur remercie les relecteurs de la revue pour de nombreuses suggestions d'amélioration.

\section{Références}

Balesdent J, Arrouays D. 1999. An estimate of the net annual carbon storage in French soils induced by land use change from 1900 to 1999. CR Acad Agri 85: 265-277.

Bouman BAM, Lampayan RM, Tuong TP. 2007. Water management in irrigated rice: coping with water scarcity. Los Baños (Laguna): IRRI, 54 p. Available from http://books.irri.org/getpdf.htm?book= 9789712202193. Site consulté le 28-07-2017.

Caron P. 2016. Climate-smart agriculture : émergence d'un concept, mise en politique, mise en sciences et controverses. Natures, Sciences, Sociétés 24(2): 147-150. Disponible sur https://doi.org/ $10.1051 / \mathrm{nss} / 2016012$. Site consulté le 28-07-2017.

Caron P, Treyer S. 2015. L'agriculture climato-intelligente et les arènes de la négociation internationale sur le changement climatique. In: Torquebiau $\mathrm{E}$, ed. Changement climatique et agricultures du monde. Versailles: Quæ, pp. 303-313.

FAO. 2010. "Climate-Smart" Agriculture - Policies, Practices and Financing for Food Security, Adaptation and Mitigation. Available from http://www.fao.org/docrep/013/i1881e/i1881e00.htm. Site consulté le 28-07-2017.

FAO. 2011. Pour une agriculture intelligente face au climat Politiques, pratiques et financements en matière de sécurité alimentaire, d'atténuation et d'adaptation. Disponible sur http:// www.fao.org/docrep/013/i1881e/i1881e00.htm. Site consulté le 28-07-2017.

FAO. 2013. Climate-smart agriculture sourcebook. Rome: FAO, $570 \mathrm{p}$.

Garnett T. 2011. Where are the best opportunities for reducing greenhouse gas emissions in the food system (including the food chain)? Food Policy 36: S23-S32.

Giller KE, Andersson JA, Corbeels M, Kirkegaard J, Mortensen D, Erenstein O, et al. 2015. Beyond conservation agriculture. Frontiers in plant science 6: 870 .

Grau R, Kuemmerle T, Macchi L. 2013. Beyond "land sparing versus land sharing": environmental heterogeneity, globalization and the balance between agricultural production and nature conservation. Curr Opin Environ Sustain 5: 477-483.

Harvey CA, Chacón M, Donatti CI, Garen E, Hannah L, Andrade A, et al. 2014. Climate-smart landscapes: opportunities and challenges for integrating adaptation and mitigation in tropical agriculture. Conservation Letters 7(2): 77-90.

Horlings LG, Marsden TK. 2011. Towards the real green revolution? Exploring the conceptual dimensions of a new ecological modernisation of agriculture that could "feed the world". Global Environmental Change 21(2): 441-452.

IPCC. 2014. Summary for Policymakers. In: Edenhofer O, PichsMadruga R, Sokona Y, Farahani E, Kadner S, Seyboth K, et al., eds. Climate Change 2014: mitigation of climate change. Contribution of working group III to the Fifth Assessment Report of the Intergovernmental Panel on Climate Change. Cambridge, United Kingdom and New York (USA): Cambridge University Press.

Joulian C, Escoffier S, Le Mer J, Neue HU, Roger PA. 1997. Populations and potential activities of methanogens and methanotrophs in rice fields: relations with soil properties. Eur J Soil Biol 33: $105-116$

Lal R. 2016. Beyond COP 21: Potential and challenges of the " 4 per Thousand" initiative. Journal of Soil and Water Conservation 71 (1): 20A-25A. DOI: 10.2489/jswc.71.1.20A.

Le Quéré C, Moriarty R, Andrew RM, Peters GP, Ciais P, Friedlingstein P, et al. 2015. Global carbon budget 2014. Earth System Science Data 7(1): 47-85.

Lipper L, Thornton P, Campbell BM, Baedeker T, Braimoh A, Bwalya M, et al. 2014. Climate-smart agriculture for food security. Nature climate change 4 : 1068-1072. Available from https://doi.org/ 10.1038/NCLIMATE2437.

Minang PA, van Noordwijk M, Freeman OE, Mbow C, de Leeuw J, Catacutan D. 2015. Climate-smart landscapes: multifunctionality in Practice. Nairobi (Kenya): World Agroforestry Centre (ICRAF), 404 p.

Nitschke CR, Innes JL. 2008. Integrating climate change into forest management in South-Central British Columbia: an assessment of landscape vulnerability and development of a climatesmart framework. Forest Ecology and Management 256(3): 313-327.

Papy F. 2016. Les agricultures du monde face au dérèglement du climat. Courrier de l'environnement de l'INRA 66: 25-33.

Perfecto I, Vandermeer JH, Wright AL. 2009. Nature's matrix: linking agriculture, conservation and food sovereignty. London: Earthscan, $242 \mathrm{p}$.

Powlson DS, Stirling CM, Thierfelder C, White RP, Jat ML. 2016. Does conservation agriculture deliver climate change mitigation through soil carbon sequestration in tropical agroecosystems? Agriculture, Ecosystems \& Environment 220: 164-174.

Reij C, Tappan G, Smale M. 2009. Agro-environmental transformation in the Sahel: another kind of "Green Revolution". IFPRI Discussion Paper 00914. Washington DC: International Food Policy Research Institute. Available from http://www.ifpri.org/ book-5826/millionsfed/cases/innovation. Site consulté le 28-072017.

Saj S, Torquebiau E, Hainzelin E, Pagès J, Maraux F. 2017. The way forward: an agroecological perspective for Climate-Smart Agriculture. Agriculture, Ecosystems and Environment 250: 20-24. DOI: 10.1016/j.agee.2017.09.003.

Saunois M, Jackson RB, Bousquet P, Poulter B, Canadell JG. 2016. The growing role of methane in anthropogenic climate change. Environmental Research Letters 11(12): 120207.

Sommer R, Bossio D. 2014. Dynamics and climate change mitigation potential of soil organic carbon sequestration. Journal of Environmental Management 144: 83-87.

Tatsidjodoung P, Dabat MH, Blin J. 2012. Insights into biofuel development in Burkina Faso: potential and strategies for 
sustainable energy policies. Renewable and Sustainable Energy Reviews 16(7): 5319-5330.

Tissier J, Grosclaude JY, 2015. Que penser de l'agriculture climatointelligente ? In: Torquebiau E, ed. Changement climatique et agricultures du monde. Versailles: Quæ, pp. 291-302.

Vayssières J, Assouma MH, Lecomte P, Hiernaux P, Bourgoin J, Jankowski F, et al. 2017. L'élevage au cœur de paysages
« climato-intelligents » en Afrique de l'Ouest. In: Caron P, Valette E, Wassenaar T, Coppens d'Eeckenbrugge G, Papazian V, eds. Des territoires vivants pour transformer le monde. Versailles: Quae, pp. 114-120.

Wezel A, Bellon S, Doré T, Francis C, Vallod D, David C. 2009. Agroecology as a science, a movement and a practice. A review. Agronomy for Sustainable Development 29: 503-515.

Citation de l'article : Torquebiau E. 2017. Climate-smart agriculture : pour une agriculture climato-compatible. Cah. Agric. 26: 66001. 